\title{
Application of Mathematical Group Concept to Human Perceptual Systems, Visual and Auditory ${ }^{1)}$
}

\author{
By Seizо Онв \\ Nippon University, Tokyo
}

The algebraic theory of groups is, as is well known, one of the most fundamental topics of modern mathematics. It has also very important applications outside of mathematics proper, especially in crystallography and recently in quantum mechanics. Moreover, it has already been introduced into the theory of perception by the so-called cybernetists $[1,11,15]$. As far as I am aware, this is an application of group theory as the theory of transformation groups and their invariants to a theoretical construction of a dynamic model for certain perceptual constancies. An entirely different type of application of group theory will be developed in this paper. It is not such a particular application of group theory to some perceptual processes, as is the case in cybernetics; rather it is an application of the mathematical group concept as such to the fundamental structure of human perceptual systems, visual and auditory. In the following I will apply the mathematical concept of group first to the system of color vision, second to the system of musical harmony, and

1) This research is financially supported by a Rockefeller Fellowship for 1956. Although the main ideas of this paper are already clear in the author's French communication at the XIth International Congress of Philosophy, Brussels, 1953 [7], as well as in his original Japanese publication in 1955 [8], the final form of this paper could only be accomplished through his personal contact with American specialists such as Professors N. Rashevsky and H. D. Landahl in Chicago, and Professor S. S. Stevens and Dr. E. H. Galanter at Harvard, and lastly by the kind help of Mr. Richard Klein, Boston University. 
third to the system of vowel perception.

Before we proceed to this task, however, a brief explanation of the mathematical group concept may be given in terms of a familiar example, i. e. the additive group of all integers including zero. The set of all integers including zero: $\{\cdots,-3,-2,-1,0,1,2,3, \cdots\}$ forms a group in the following sense :

(a) For all elements of the set an additive operation $(a+b)$ is defined such that the result of this operation between any two elements of the set is again an element of the set, as the sum of any two integers is again an integer.

(b) This operation satisfies the associative law of algebra, that is to say, for any three elements $a, b, c$ of the set the following relation always holds: $(a+b)+c=a+(b+c)$.

(c) There is in the set a special element 0 , called the identity element of the group, such that for any element a of the set an additive operation with 0 turns a again into itself, so that $a+0=a$.

(d) There is in the set for each element a another element -a, called the inverse element of a, such that an operation between these two elements always gives the identity element of the group, so that at $(-a)=0$. The identity element is inverse to itself : $0+0=0$.

These four conditions must be satisfied by any group in the proper sense $[14]^{2)}$. If only the first two conditions are satisfied, then we have a semi-group, e. g. the set of all positive integers.

\section{The Group Structure of Color Vision}

It is well known that, in spite of the discontinuous arrangement of the

2) If, moreover, the additive operation of the group satisfies the commutative law of algebra, so that for any two of its elements $a, b$ always $a+b=$ $\mathrm{b}+\mathrm{a}$, the group is called a commutative or Abelian group. This is obviously the case not only with the above example but also, theoretically at least, with all three following cases of application, though we will not mention it specifically 
primary photorecepters on the retina, our visual perception is continuous throughout. Moreover, the so-called pointillists of French post-impressionism have even succeeded in a way to create a vivid and fresh effect of color by juxtaposing colored points on the canvas. In this sense, each perceptual aggregate of our color vision already possesses essentially the same structure as that of a mathematical set in its intuitive Cantorian definition: "Any collection into a whole, of definite, well distinguished objects of our perception or our thought, which are called the 'elements' of the set" [2, p. 282]. But, the set of all elemental color perceptions has, furthermore, the structure of a mathematical group, essentially satisfying the above mentioned four conditions of a group.

We all know, on the one hand, that the white sunlight can be decomposed into a series of six principal colors: violet-blue-green-yelloworange-red; and this corresponds to a very small section of wavelength from about 400 to $700 \mathrm{~m} \mu$ in the spectral system of all electromagnetic waves, the prismatic "color band" of visible light. On the other hand, everyone knows that these same six colors as perceptual elements of our color vision do not form a series but a closed system, connecting both ends of the color band, violet and red, with a new intermediate color, purple. We can represent it as a "color circle" with neutral white in the center, as in Fig. 1. This is the fundamental fact which lies at the core of all geometrical representations of our sensory color

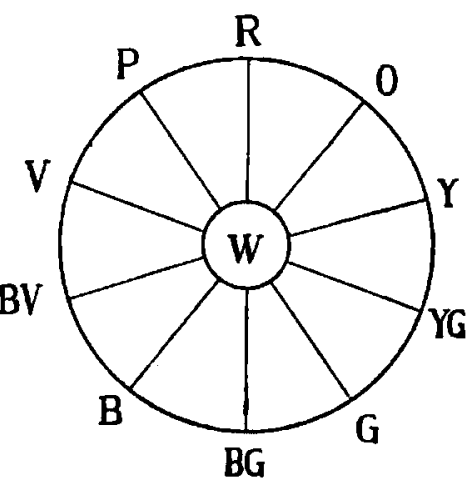

Fig. 1 system. Yet few people seem to have noticed that this circle of colors constitutes a group in the mathematical sense, having color mixing as an additive operation, neutral white as the identity element, and complementary colors as the inverse elements. ${ }^{3)}$

3) Meanwhile, it has become clear to the present author that Professor S. S. Stevens, too, has already had a similar idea, and he has also called my attention to C. J. Keyser's Mathematical Philosophy-A Study of Fate and Freedom, New York: E. P. Dutton \& Co., 1922, in which the following very 
If we disregard various technical problems of color mixing and focus our attention primarily on hues, the demonstration of the group structure of the color circle can be made rather simply as follows :

(a) The operation of color mixing plays the same role in the color group as does addition in the group of all integers including zero. For, the mixture of any two colors obviously yields a color, as the sum of any two integers is again an integer. Some of the mixtures, to be sure, simply produce white, which is, however, to be regarded as a special color, the "zero color". The simplest way of finding experimental confirmation might be the method of combining spectral lights. In this type of color mixing, "additive color mixing", the brightness increases. But, if we keep our attention only upon hues as such, we can obtain, through suitable mixing of three primary colors : red, green, and violet, all possible hues including non-prismatic hues, namely the purple series which is lacking in the spectral system. This means, we obtain all the just distinguishable elements on the periphery of the color circle. If we further consider all these primary and secondary hues as normalized in a suitable way, then experimental confirmation may not be so difficult. Any just noticeable hue must find its place in such a complete system of normalized hues and every element in it must be obtained by a suitable combination of its two elements, although it would be, of course, impossible to extend this procedure to the extreme cases of two just distinguishable hues.

(b) The operation of color mixing in the sense of addition of hues satisfies the associative law of algebra, just as does arithmetical addition. For example, in the case of mixing light beams of three different colors: red, yellow and blue, whether red and yellow or yellow and blue are

suggestive words are to be found on p. 208: "As the combination of any two color sensations is a color sensation, S11 [the system of mixing of color sensations] has, you see, the group property [the closure property]. Is it a group? Evidently condition (b) [the associative law] is satisfied. Are conditions (c) and (d) [the existence of identity and inverse elements] also satisfied ?" 
mixed first, is obviously irrelevant for the final result, as may be indicated schematically: $(\mathrm{R}+\mathrm{Y})+\mathrm{B}=\mathrm{R}+(\mathrm{Y}+\mathrm{B})$.

(c) The neutral white is a special color which does not change the hue of any other color with which it is mixed, just as the special number 0 does not change the value of any other number with which it is added. Therefore neutral white is the identity element of the color group. For instance, if we mix red light with white light or if we put red paint on white paper, the redness certainly decreases. But this is due only to a decrease of saturation, not a change of hue. In this sense, pink is an unsaturated red, and as far as hue is concerned, red mixed with white remains red, schematically: $R+W=R$.

(d) To every color there is a complementary color which, when mixed, annihilates the hue of the original and turns it into colorless white, $i$. e. into the identity element of the color group, just as two integers of the same numeral but of opposite signs, when added, yield 0 , the identity element of the integer group. Thus the complementary color is the inverse element of the color group; for example red is complementary to bluish green, yellow to bluish violet, etc. In the color circle complementary colors are usually located at opposite ends of the diagonals, and white at the center is inverse to itself. Theoretically this is a well established fact, although technical difficulties might still remain in determining the "complementary equivalents", viz. the exact relative values of complementary colors to each other. Primarily it depends upon the methods of color mixing, and in the case of "subtractive color mixing", which is practically very important in various forms of pigment mixing, even the resulting neutral color is not white but gray.

Now it should be quite clear that the system of our elemental color perceptions possesses essentially the same structure as a mathematical group, such as the additive group of all integers including zero. But it might be of some interest to add that the series of neutral colors from white to black, constituting the vertical axis of the usual solid representation of our whole perceptual color system, forms no group in the 
proper sense but a semi-group, just as in the case of the set of all positive integers, a semigroup which satisfies only the first two conditions of a group. In fact, we have in the animal world some effectively working systems of colorless vision.

\section{The Group Structure of Musical Intervals}

It is a familiar fact that the "major triad", the most popular threenoted chord, Do-Mi-So, for instance, forms in our usual experience an unseparated, harmonious whole, although it is easily analyzable into three distinct elemental tones by trained ears. Even a single musical tone, the second lowest $\mathrm{C}$ of an ordinary piano, for example, consists in reality of a fundamental tone $C$ with frequency $64 \sim$ per sec. and of a theoretically infinite series of its overtones, though only some of its earliest members can be made discriminable. Another'familiar fact: a well trained listener to an orchestra is always capable of noticing any slight defect in any of its numerous instruments, yet thoroughly enjoying the aesthetic beauty of the harmonious whole. All these facts clearly indicate that each aggregate of our musical perception has in principle the same structure of a mathematical set, as is given in its intuitive Cantorian defnition. But, the system of musical intervals based upon the usual tone scale has, furthermore, the structure of a mathematical group, satisfying all its four conditions-to be sure, a particular kind of group in this case.

As is well known, the usual musical scale of one octave can be developed both upward and downward to the threshholds of hearing, repeating the same scale of seven different tone qualities: C, D, E, F, G, $A, B$, so that every eighth note represents essentially the same tone quality as the first one, just one octave above or below. Thus all C's have a certain tone quality in common. This fact clearly suggests that a certain peculiar circularity underlies the whole system of musical perception. However, if we turn our attention to the elemental percep- 
tions of musical intervals, this peculiar circularity can be structurally clarified by means of the number-theoretic concept of a residual system of natural numbers.

In the usual, equal-tempered tone scale, as in Fig. 2, we have

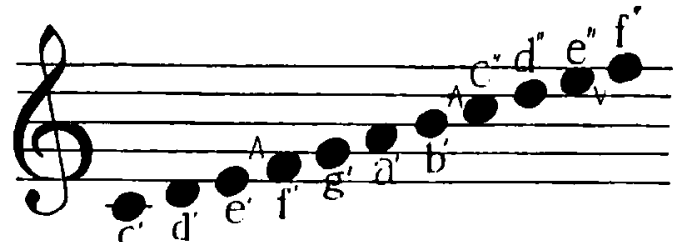
Fig. 2 in principle thirteen elemental intervals of different halftones: unison (0 halftone), minor 2nd ( 1 halftone), major 2nd ( 2 halftones), minor 3rd ( 3 halftones), major 3 rd ( 4 halftones), perfect 4 th ( 5 halftones), augmented 4th (6 halftones) equal diminished 5th (6 halftones), perfect 5 th ( 7 halftones), minor 6 th ( 8 halftones), major 6 th (9 halftones), minor 7 th (10 halftones), major 7 th (11 halftones), and octave (12 halftones). But, since an octave is again an interval between two tones of the same quality, e. g. between two C's, and accordingly its harmonic quality is essentially similar to that of unison, it can be considered as qualitatively reducible to the latter. By the same token, a major 9th is qualitatively reducible to a major $2 \mathrm{nd}$, both being intervals, say, between a $\mathrm{C}$ and a $\mathrm{D}$; likewise a major 10 th is reducible to a major $3 \mathrm{rd}$, both being intervals, say, between a $\mathrm{C}$ and a $\mathrm{E}$, etc. In fact, they are usually called "octave and a major 2nd", "octave and a major 3rd", and so forth. Thus every interval larger than a major 7th (11 halftones) can be reduced, to the corresponding one smaller than an octave ( 12 halftones), and we have actually to consider only twelve elemental intervals of different halftones from 0 to 11 .

This particular situation regarding the system of twelve elemental intervals is exactly the same as is found in the mathematical concept of a cyclic group of residue classes of natural numbers of order twelve. More specifically, natural numbers can be classified into twelve classes according to the residues they give when divided by $12:\{0,1,2,3,4,5$, $6,7,8,9,10,11\}(\bmod 12)$, and members of one and the same class are said to be congruent modulo 12 , e. g. $0 \equiv 12 \equiv 24 \cdots(\bmod 12), 1 \equiv 13 \equiv 25 \cdots$ $(\bmod 12), 2 \equiv 14 \equiv 26 \cdots(\bmod 12)$, etc., just as in the cipher system of our ordinary. clock. The system of these twelve residue classes represented 
by their residue numbers corresponds one to one to the system of twelve elemental intervals represented by the numbers of their halftones from 0 to 11. More than this, since the addition of intervals, in the sense of arithmetical addition of their halftone numbers, functions exactly in the same way as the addition of residue classes, as is apparent for example in the following parallel:

perfect 5 th + major 6 th $=$ major 10 th $\equiv$ "octave and a major 3 rd"

$$
7+9=16 \equiv 4(\bmod 12) \text {, }
$$

both systems are mathematically identical. Hence their common group structure can be shown as follows:

(a) Addition of any two elements of the system yields again an element of the system by congruence modulo 12 , e. g. $7+9=16 \equiv 4(\bmod 12)$. This implies, for the interval system, the qualitative reducibility of the intervals larger than a major 7 th ( 11 halftones) to the one smaller than an octave (12 halftones).

(b) Such an addition satisfies the associative law of algebra, just as an ordinary addition; for the addition of three intervals, it is also irrevalent which two of the three are first associated.

(c) There is in the system a special element 0 , the identity element of the group, e. g. $5+0=5$. This means, in the interval system, a unison is an interval of 0 halftone and adds nothing to another with which it is added.

(d) To every element of the system there is an inverse element; for instance 7 is inverse to 5 because $7+5=12 \equiv 0(\bmod 12)$. Translated into terms of musical intervals, this example asserts: perfect 5 th + perfect 4 th $=$ octave $\equiv$ unison. In this sense, a perfect 5 th and a perfect 4 th are called "complementary intervals", likewise a diminished 5th and an augmented 4th (both 6 halftones), a major 3rd (4 halftones) and a minor 6th ( 8 halftones), etc. They are, as is well known, very important relations ("inversions") in the theory of musical composition.

Thus, the system of musical intervals constitutes a mathematical group in the same sense as the system of residue classes of natural numbers. 
And the same can be shown more easily with respect to the "whole tone scale", which has only six whole tone steps instead of the usual five whole tone and two halftone steps. In this case, since all irregularities such as the distinction between major and minor intervals disappear, we have to consider only six elemental intervals and accordingly to apply to this simplified system of intervals the cyclic group of natural numbers of order 6 , instead of 12 . In this way the entire procedure becomes much simpler, but as a consequence we will depart a little from the realities of our common musical sense of harmony. In any case, the intrinsic circularity of our musical perception can now be given a clear structural foundation.

\section{The Group Structure of Spoken Vowels}

A spoken word, for example "America", is usually perceived as a unified whole, but it can be decomposed into four distinct syllables: A-me-ri-ca. Each of these four syllables forms a well distinguishable perceptual unit, having a vowel as its nucleus. In this sense, a perceived word has again the structure of a mathematical set in its intuitive Cantorian definition. But, whether the system of all the vowels, which are the very nuclei of spoken words, has furthermore essentially the structure of a mathematical group, is an entirely new and interesting problem which now faces us.

Roughly schematized, the system of spoken vowels forms a characteristic constellation of a double triangle as Fig. 3. This is a point where most of the modern phonetic theories converge. Yet few people seem to have imagined that this popular "triangle of vowels" possesses a structure at least very similar to that of a mathematical group. Everyone knows, however, that all the vowels used in various languages are either one of the five fundamental vowels: A, E, I, O, U, or some possible mixtures of them. Let us take a few familiar examples. Even two of these fundamental vowels, $O$ and $E$, can be regarded as a mixed vow- 
el of $A$ and $U$, and of $A$ and $I$ respectively, as French formations $a u$ (o) and $a i$ (e) suggest. German Umlaut formation is obviously nothing but a kind of vowel mixing in our own mouth : $\ddot{o}$ is a mixture of $\mathrm{O}$ and $\mathrm{E}, \ddot{u}$ a mixture of $\mathrm{U}$ and $\mathrm{I}$, and $\ddot{a}$ a mixture of $\mathrm{A}$ and $\mathrm{E}$. The last example is very close to English $a$ in cat which is also a mixture of $\mathrm{A}$ and E. Another English peculiarity $\partial$ provides us with an important mixture example of three fundamental vowels : $a$ in alone, $e$ in system, and $o$ in gallop are namely neither $\mathrm{A}$ nor $\mathrm{E}$ nor $\mathrm{O}$, but something in

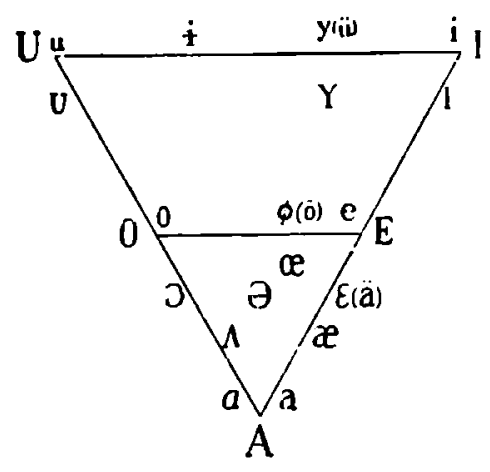

Fig. 3 between all three. Accordingly, $ə$ can be regarded as a mixture of $A, E$ and $O$, and is, in fact, called a "neutral vowel", although it is really neutral only within the AEO subsystem. In the AIU system as a whole it is simply an "unsaturated" A. A certain analogy with the color system is therefore at hand.

Keeping all these data in mind, let us first examine the smaller triangle AEO of Fig. 3 with neutral vowel $a$ in the middle. All the phonetic vowels such as $a, a, æ, \varepsilon, e, \phi, \propto, 0,0, \Lambda, \partial$, etc., which are closely affiliated with $A, E$, and $O$ in one way or another, can approximately be located in it. However, since the phonetic qualities even of these standardized vowels sensitively diverge in various languages, we are compelled to rely primarily upon a method of schematization. For the sake of convenience, we assume in Fig. 4 triangle AEO to be a regular triangle, precisely forming the lowest quarter of the larger regular triangle AIU of Fig. 3, and we locate in it, instead of eleven abovementioned phonetic vowels, first only six vowel points: $\mathrm{O},\left(\begin{array}{l}\mathrm{O} \\ \mathrm{A}\end{array}\right), \mathrm{A},\left(\begin{array}{l}\mathrm{E} \\ \mathrm{A}\end{array}\right), \mathrm{E},\left(\begin{array}{l}\mathrm{O} \\ \mathrm{E}\end{array}\right)$ on the periphery and a $ə$-circle in the middle, just inscribed in triangle $\left(\begin{array}{l}\mathrm{O} \\ \mathrm{A}\end{array}\right)\left(\begin{array}{l}\mathrm{E} \\ \mathrm{A}\end{array}\right)\left(\begin{array}{l}\mathrm{O} \\ \mathrm{E}\end{array}\right)$ whose center is to be represented by (AEO). We further assume that vowel. mixing, as we have in German Umlaut formation can be represented by a straight line connecting

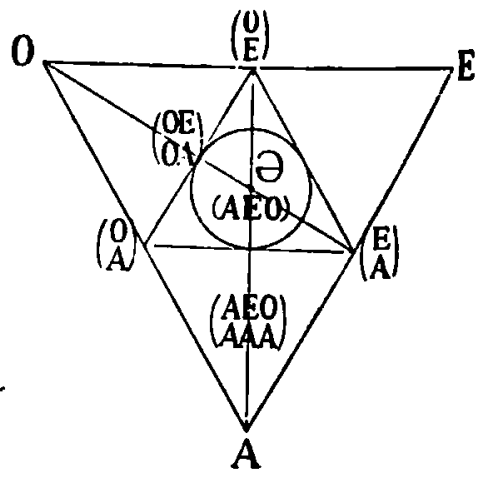

Fig. 4 
two vowel points and the resulting mixed vowel by the mid-point of this line, whereby we restrict the mixing ratio in principle to the simplest case of $1: 1$. Then, we can easily show that this AEO subsystem of vowels has a structure which essentially satisfies the four conditions of a mathematical group, as follows:

(a) The mixture of any two vowels of this AEO system belongs again to this system. As is illustrated in Fig. 4, the mid-point of any straight line between any two vowel points can never fall outside of triangle $\mathrm{AEO}$, although it would be practically meaningless to extend this procedure to the extreme cases of mixing two just distinguishable vowels. If we schematize; according to our assumptions, in the following intuitive way e. $g$. the mixing of $E$ and $\left(\begin{array}{l}E \\ A\end{array}\right)$ by

$$
E+\left(\begin{array}{l}
E \\
A
\end{array}\right)=\left(\begin{array}{l}
E \\
E
\end{array}\right)+\left(\begin{array}{c}
E \\
A
\end{array}\right)=\left(\begin{array}{c}
E E \\
E A
\end{array}\right)
$$

the result indicates, when applied to Fig. 4, the mid-point between $\mathrm{E}$ and $\left(\begin{array}{l}E \\ A\end{array}\right)$. This might still correspond to phonetic sign $\varepsilon$, but a further mixture of this vowel with E could have only a theoretical meaning, if at all.

(b) Such an operation of vowel mixing satisfies the associative law of algebra. If we remember that also in the case of three vowels the mixing ratio should remain $1: 1: 1$, then this operation evidently satisfies the associative law, e. g.

$$
(\mathrm{A}+\mathrm{E})+\mathrm{O}=(\mathrm{AEO})=\mathrm{A}+(\mathrm{E}+\mathrm{O}) .
$$

(c) In this AEO system there is a special element, neutral vowel $ə$, which plays the role of the identity element of a group. If we schematize e. $g$. the mixing of $A$ with neutral vowel a by

$$
\mathrm{A}+\partial=(\mathrm{AAA})+(\mathrm{AEO})=\left(\begin{array}{l}
\mathrm{AEO} \\
\mathrm{AAA}
\end{array}\right) \text {, }
$$

the resulting formula indicates, when applied to Fig. 4, the mid-point between $\mathrm{A}$ and the center of the a-circle. Due to our assumed geometrical construction, that is the point which divides perpendicular $\mathrm{A}\left(\begin{array}{l}\mathrm{O} \\ \mathrm{E}\end{array}\right)$ at the ratio of $1: 2$, and this corresponds exactly to what $\left(\begin{array}{c}A E O \\ A A A\end{array}\right)$ sche- 
matically indicates, especially when it is analyzed into $\left(\begin{array}{c}A A \\ A A\end{array}\right)+\left(\begin{array}{l}O \\ E\end{array}\right)$. Since this vowel formula $\left(\begin{array}{c}A E O \\ A A A\end{array}\right)$ contains, besides the neutralizing AEOcompound, nothing but three A-elements, it is to be interpreted within this AEO subsystem as an "unsaturated" A. Therefore we can say: neutral vowel $ə$, when mixed with another vowel of this AEO system, e. g. with A, makes the A-quality less articulated but does not change the A-quality as such, just as neutral white does not change the hue of another color with which it is mixed.

(d) In this AEO system to each vowel can be assigned another vowel, which plays the role of the inverse element of a group. Theoretically, when we draw in Fig. 4 from any vowel point through the center of the ə-circle and reach the other side of triangle AEO, we get the inverse vowel point. For example, from $O$ we reach $\left(\begin{array}{c}E \\ A\end{array}\right)$ and their mutually inverse relation can be shown schematically by the following formula:

$$
\mathrm{O}+\left(\begin{array}{l}
\mathrm{A} \\
\mathrm{E}
\end{array}\right)=\left(\begin{array}{l}
\mathrm{O} \\
\mathrm{O}
\end{array}\right)+\left(\begin{array}{l}
\mathrm{E} \\
\mathrm{A}
\end{array}\right)=\left(\begin{array}{l}
\mathrm{OE} \\
\mathrm{OA}
\end{array}\right) \cong(\mathrm{AEO})
$$

The formula $\left(\begin{array}{c}O E \\ O A\end{array}\right)$ indicates, when applied to Fig. 4, the mid-point between $O$ and $\left(\begin{array}{c}E \\ A\end{array}\right)$, which lies just on the periphery of our supposed area of AEO-neutrality, and, due to our assumed geometrical construction, $\left(\begin{array}{l}E \\ A\end{array}\right)$ is the only possible vowel point which is inverse to $O$ by our assumed equivalent mixing, because for any other point of line AE the mid-point of the line which connects it with $O$ falls neither on nor within the a-circle. On the contrary, with respect to $\left(\begin{array}{l}E \\ A\end{array}\right)$ we can find many other such points in the neighborhood of $O$. This curious, somewhat perplexing situation, however, quite conforms to the theoretical status of these two vowels. Namely, while $\left(\begin{array}{c}E \\ A\end{array}\right)$ is a mixed vowel and as such more easily neutralizable, $O$ is a pure, fundamental vowel within AEO subsystem and as such harder to neutralize. In the end, all such irregularities are nothing but the natural consequences of the assumed triangular construction of the vowel system, in this case the AEO subsystem, and of our equally assumed area extention of neutral vowel $\partial$-two fundamental assumptions which are, however, essentially based upon the real status 
of spoken vowels.

Certainly, all these mathematical manipulations would have no meaning at all, if not based upon realities. But, if it is sufficiently supported by empirical data, as the present author believes to be the case at least with the above AEO subsystem, then it will not only be capable of providing experimental research with useful suggestions but also be helpful for possible future improvement of vowel pronunciation in various language systems. Generally speaking, our vowel system as a whole, as it is presented in Fig. 3, seems to be not as empirically secure as the above AEO subsystem. The most important defect in this respect is undoubtedly the fact that there yet exists no commonly acknowledged neutral element in AIU system comparable to the neutral vowel $\partial$ in the AEO system. But, once such a corresponding neutral element is assumed to exist in the middle of triangle AIU, as is indicated by a dotted circle in Fig. 5, our argument for triangle AEO can easily be applied mutatis mutandis to triangle AIU, because the latter is assumed to be geometrically similar to the former, the AEO system thus turning out to be a sub-group of the whole vowel group. Hence, the problem of group structure for the whole vowel system revolves now around the question of the existence of a neutral area within triangle AIU.

The question which now confronts us is mainly an empirical one. Let us first try to examine it via our own experience. Try, for instance, to pronounce $i$ with the lip position for o or o with the lip position for $i$, and you will get only a burst of air, just as in the case of an isolated ch-pronunciation in German. With $u$ and e, or with $a$ and $y$, you will have much the same experience. After you have succeeded to standardize this way of voiceless pronunciation, try to do it with the lip position for A. Then you will find that the simple burst of air now gets a tinge of A-quality, just as does ch in German words such as Bach, Dach, etc. Actually it will come out to something like an A spoken in a whisper.

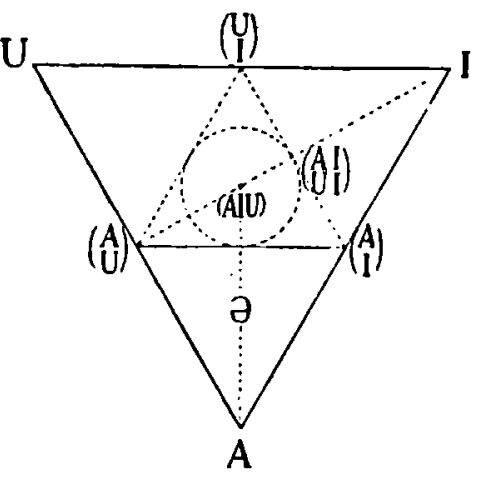

Fig. 5 
With $U, O, I$, or E, you will have a similar experience.

Now, let us try to interpret these facts with our formulas. Pairs of vowels : o-i, u-e, a-y, approximately correspond to pairs of vowel points: $\left(\begin{array}{l}A \\ U\end{array}\right)-I, U-\left(\begin{array}{l}A \\ I\end{array}\right), A-\left(\begin{array}{l}U \\ I\end{array}\right)$ in Fig. 5 respectively, which are, by analogy with the AEO system in Fig. 4, supposed to be pairs of inverse elements in the AIU system. Hence no positive vowels can be produced by mixing such pairs. They annihilate each other's vowel qualities, for example

$$
\mathrm{O}+\mathrm{I}=\left(\begin{array}{l}
\mathrm{A} \\
\mathrm{U}
\end{array}\right)+\left(\begin{array}{l}
\mathrm{I} \\
\mathrm{I}
\end{array}\right)=\left(\begin{array}{l}
\mathrm{AI} \\
\mathrm{UI}
\end{array}\right) \cong(\mathrm{AIU}) \text {, }
$$

where (AIU) represents the supposed neutrality center of the AIU system. That such a supposed "zero vowel", so to speak, when mixed with a plain vowel such as A, easily takes on a tinge of A-quality, can also be shown schematically :

$$
\mathrm{A}+(\mathrm{AIU})=(\mathrm{AAA})+(\mathrm{AIU})=\left(\begin{array}{l}
\mathrm{AAA} \\
\mathrm{AI} U
\end{array}\right)=(\mathrm{AEO})
$$

Here (AEO) is the center of the a-circle in triangle AEO. This is the reason why neutral vowel $\partial$ of the AEO subsystem must be simply an "unsaturated" A in the AIU system. In fact, $\partial$ always sounds something like an unarticulated $\mathrm{A}$.

After what has been briefly explained above, an absolutely neutral vowel, as it is suppoed to be located in the middle of the AIU vowel system, must be a kind of "white noise" as physicists call it, to which "voiceless fricatives", "voiceless aspirates", etc., as linguists call them, are certainly congenial [4]. The spectrographic representation of speech sound assures us also of the existence of such areas which rather serve as the neutral background for making visible patterns of various vowel qualities [12]. It is quite understandable, however, that a vowel triangle in the usual linguistic-phonetic version does not contain any indication regarding such an absolutely neutral area within it, because such a "zero vowel" is "actually not a vowel in any sense and has no positive value for vowel pronunciation. On the contrary, some of the physicophysiological researches on speech sound, which are naturally concerned with a broader aspect of vowels, indicate very clearly the existence 
of such an absolutely neutral area within the vowel system itself $[3,5]$. Since the technique of tone-preservation, -representation, and -reproduction is becoming more and more elaborate and efficient, it is not impossible that our arguments concerning the group structure of the human vowel system will be in many ways re-examined and essentially verified by refined methods of phono-technical experiment in the near future.

For such purposes too, the following remarks might be of some use. The one concerns the double closure of the vowel triangle. The other concerns the fundamental regularity of this construction.

1. The human vowel system is apparently a by-product of animal evolution, especially mammalian evolution. Animal voices, as means of communication, naturally require more and more clear articulation. Two conceivable processes of vowel evolution in the animal world would be the following, as indicated in Fig. 6 .

In the case of the process : I-IIa-III, the double closure of the vowel triangle is almost self-evident, because in this case $A ; E$, and $O$ are supposed to be still indiscriminable on the second stage. But, even the other process: I-IIb-III does not contradict the dou-

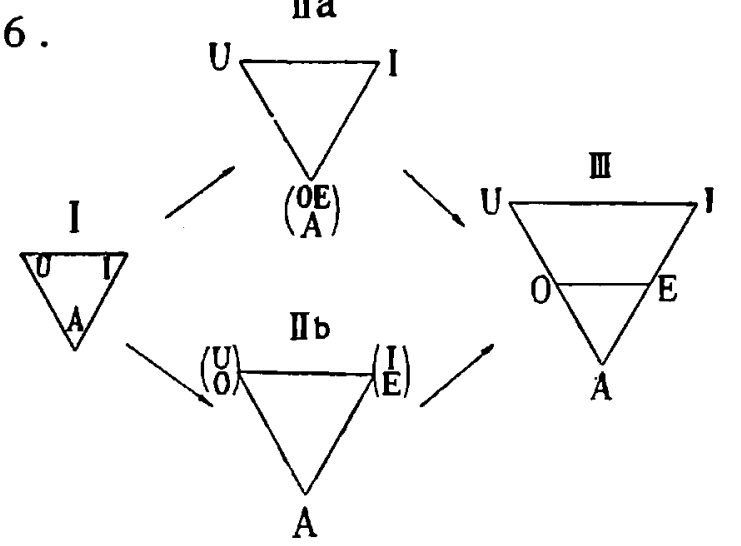

Fig. 6

ble closure of the vowel triangle, for in IIb too the AEO system is potentially present. Moreover, the fact that in Sanscrit three simple vowels $A, E$ and $O$ have been reduced to one simple vowel $A$, seems to strongly testify to the original affinity of these three vowels [13]; and that in English the actual pronunciation of $a$ covers almost the whole range from $O$ to $E$, is also certainly not an unfavorable fact for the justification of the AEO-closure.

2. According to the acoustic experiment of W. Köhler [6], in the frequency series of pure physical tones, marked resemblances with $U$-, O-, A-, E-, I- vowe1 qualities are statistically confirmable in tones of about 
$\mathrm{c}^{1}, \mathrm{c}^{2}, \mathrm{c}^{3}, \mathrm{c}^{4}, \mathrm{c}^{5}$ frequencies respectively, with a spacing of approximately one octave apart. Remarkably enough, nowhere in this series of pure physical tones were found $\ddot{o}$-and $\ddot{u}$-resemblances, the two vowel qualities particularly, which close the vowel system to a double triangle, while $\ddot{a}$-resemblance is noticeable at its proper place between $\mathrm{A}$ and $\mathrm{E}$. This interesting result, though still debatable, attracts our attention, first, because of its striking similarity to the relation between the physical "color band" and the psychological "color circle", and, second, because of its regularity of spacing in comparison with the irregular spacing of the spectral color series. On the whole, Köhler's experiment, too, seems in principle to justify the double regular triangle constellation of the five fundamental vowels, although, to be sure, such a theoretically simplified construction as ours can only serve us as a mathematical model of the real system of spoken vowels.

\section{SUMMARY}

I. The system of color vision forms a group, having color mixing as an additive operation, neutral white as the identity element, and complementary colors as the inverse elements.

II. The system of musical intervals forms a group in the same sense, as does the system of residue classes of natural numbers with respect to an additive operation by congruence modulo 12 .

III. The system of spoken vowels is supposed to form a double group. The actual demonstration is only made with respect to its subsystem, the AE.O subgroup of vowels with neutral vowel $\partial$ as the identity element; and by assuming a corresponding neutral element in the whole vowel system, the group structure of the latter is theoretically clarified.

In this paper the author is intentionally refraining himself from any philosophical argumentation. But, in other papers [especially in 9 and 10], he is trying to draw some epistemological consequences from the above developed theory of perception. 


\section{References}

[1] F. H. Allport: Theories of Perception and the Concept of Structure; New York, Wiley, 1955.

[2] G. Cantor: Gesammelte Abh. ed. by E. Zermelo; Berlin, Springer, 1932.

[3] H. Fletcher: Speech and Hearing in Communication; New York, Van Nostrand, 1953.

[4] H. A. Gleason: An Introduction to Descriptive Linguistics ; New York, Henry Holt, 1955.

[ 5 ] E. M. V. Hornbostel: Psychologie der Gehörsercheinungen ; Handbuch der Normalen u. Pathologischen Physiologie, Berlin, Springer, 1926, 11, $701-730$.

[6] W. Köhler: Akustische Untersuchungen II; Z. f. Psychol. 58 (1911), .59-140.

[7] S. Ohe: La Structure Mathématique des Sensations: Proc. 11th Intern. Congr. Phil., Brussels, 1953, 7, 91-93.

[8] S. Ohe : Kankaku no Sūgakuteki Kōzō (mathematical structure of senseperception); Tokyo, Sōbunsha Publ. Co., 1955.

[9] S. Ohe: Le Problème de la Réalité Objective et la Structure Multiple de notre Connaissance Extérieure; Revue Philosophique, Paris, 79 (1954), 416-419.

[10] S. Ohe: Die Mehrfache Struktur der Naturerkenntnis; Proc. 2nd int. Congr. of the U. I. P. S., Zurich, 1954, 3, 94-97.

[11] W. Pitts and W. S. McLulloch: How We Know Universals-The Peroeption of Auditory and Visual Forms; Bull. Math. Biophys. 9 (1947) 127-147.

[12] R. K. Potter, C. A. Kopp and H. C. Green : Visible Speech ; New York, Van Nostrand, 1947.

[13] J. Schrijnen and W. Fischer: Einführung in das Studium der Indogermanischen Sprachwissenschaft; Heidelberg, C. Winter, 1921.

[14] S. S. Stevens : Mathematics, Measurement, and Psychophysics ; Handbook of Experimental Psychology ed. by S. S. Stevens, New York, Wiley, $1951,1-49$. 
[15] N. Wiener : Cybernetics ; New York, Wiley, 1948.

(Tokyo, Nov. 3, 1956) 